\title{
VAC_23 - Optimization and validation of an alternative method of residual moisture for quality control of lyophilized Measles, Mumps and Rubella vaccine
}

Karolyne Barreto de la Torre Ruibal ${ }^{1 *}$; Ana Cláudia Bergamo ${ }^{1}$; Alan Gomes Pinto Sobrinhoㅎ ; Denivaldo Belarmino da Silva ${ }^{1}$; Simone Ferreira Rodrigues Fernandes ${ }^{1}$; Daniel da Silva Guedes Junior ${ }^{1}$.

${ }^{1}$ Fiocruz/Bio-Manguinhos.

Introduction: The determination of residual moisture is an important quality parameter for lyophilized vaccines and biopharmaceuticals, due to the water content being intrinsically related to the stability of the product. The most used technique for the determination of residual moisture of lyophiles is coulometric titration, as this technique is indicated to quantify small amounts of water. In this method, the iodine required in the reaction medium to react with water is formed from electrochemical and the electrode measures the time and current flow necessary to reach the end point of the titration. The application of the method can occur by direct transfer, in which the lyophile is crushed and poured directly into the equipment vessel, or by reconstitution, where a small volume of reconstituted lyophile is injected into the equipment. The reconstitution method has numerous advantages, such as less occupational exposure to organic solvents; decrease in solvent consumption, consequently generating less chemistry residue; shorter analysis time and reduced analysis cost as less solvent is spent.

Objective: Optimize and validate the methodology for determining residual moisture by the reconstitution method for application in the quality control of Measles, Mumps and Rubella Vaccine.

Methodology: The vial with the lyophile is reconstituted with $5 \mathrm{~mL}$ of Karl Fischer reagent and homogenized. Subsequently, a $0.5 \mathrm{~mL}$ aliquot is removed from the vial and inserted into the equipment. This procedure is done in triplicate. The calculation of residual moisture in the vial makes use of the amount of water measured by the equipment, total mass after reconstitution, theoretical mass of the lyophile and the mass injected into the vessel. Accuracy was assessed through recovery, using a water reference standard in three concentrations: high, medium and low considering specification of the vaccine. Due to the variation of the existing residual moisture between vials, precision was assessed from measurements within the same vial (intra-vial). The precision study was conducted in two days by two analysts analyzing three vials of the vaccine, each vial analyzed in quintuplicate.

Results: The accuracy showed recovery results between $97 \%-103 \%$ at concentrations $80 \%, 100 \%$ and $120 \%$. The intermediate precision showed coefficients of percentage variation (CV\%) between $0.68 \%$ and $2.49 \%$, being within the $\mathrm{CV} \%<5 \%$ acceptance criteria.

Conclusion: The validation of the methodology met the acceptance criteria. Karl Fiescher's methodology by reconstitution was able to provide numerous advantages such as reducing solvent usage and consequently the cost of analysis, less waste generation, no occupational exposure to toxic solvents and shorter intervals for cleaning and changing the reagent of the equipment, reducing the time to perform the analysis.

Keywords: Moisture; Karl-Fischer; Reconstitution 